\title{
Automatic fabrication for bridged laminated object manufacturing (LOM) process
}

\author{
Y.Y. Chiu ${ }^{\text {a } *}$, Y.S. Liao ${ }^{\text {b }}$, C.C. Hou ${ }^{\text {b }}$ \\ ${ }^{a}$ Department of Industrial Design, Oriental Institute of Technology, 58, Sec. 2, \\ Syh-Chuan Road, Baan Chyan, 220 Taipei, Taiwan, ROC \\ ${ }^{\mathrm{b}}$ Department of Mechanical Engineering, National Taiwan University, Taipei, Taiwan, ROC
}

\begin{abstract}
Laminated object manufacturing (LOM) is one of the most popular rapid prototyping (RP) techniques in terms of speed and cost effectiveness. Despite the advantages of the LOM process, some problems are yet to be overcome. One of the major difficulties encountered in the current LOM practice is the metallic part cannot be fabricated. Focusing on the drawback inhered in LOM, a new Bridge-LOM process and its associated building algorithm are proposed in this paper. The process starts with the construction of the bridge structures to link a stack of 2D geometry contours to the outer frame based on the proposed bridge building algorithm. Afterwards, laser is directed to cut along the contours, and then the upper pressing head is pushed down and the layers are bonded. Spraying adhesive on the top of bonded layers follows this. The procedures of cutting, bonding and adhesive spraying are repeated until the complete part is produced. The proposed Bridge-LOM process has overcome two problems, support and position. Regarding the function of position, in order to ensure the proper bonding treatment, as well as holding a precise position between layers, the processes from cutting to bonding in the process should maintain the same $X-Y$ position. Therefore, worries of not having a fixed positioning would then be eliminated. Regarding the function of support, bridge has taken the place of the supporting function of waste material in the current LOM process. The metallic part can be built directly based on the efficiency of on-line waste material removal in the process. From the experiments, it is verified that the proposed Bridge-LOM process and its associated building algorithm not only save laser-cutting time, the time on waste material removal is also reduced significantly. Furthermore, the metallic part and mold can be fabricated and their performances are investigated.
\end{abstract}

(c) 2003 Elsevier B.V. All rights reserved.

Keywords: Laminated object manufacturing (LOM); Bridge-LOM; Waste material removal

\section{Introduction}

"A diagram serves better than one thousand words for description, whereas a solid model serves better than one thousand diagrams for illustration." This saying shows the importance of a solid model. Nowadays, rapid prototyping (RP) can achieve this goal rapidly. The first step of the product development is to design the necessary CAD file. Next, build a prototype of the product according to the file, so as to confirm the design, revise the product, test its function and then further develop its mold. Laminated object manufacturing (LOM) process carries the advantages of using low-price materials, as well as speedy and processing, etc. It adopts the LOM process by using paper as the laminated materials. It uses the "bond-then-cut" principle. A sheet is laminated to the previously laid and bonded layers by a hot roller. The roller applies heat and pressure as it is rolling over

\footnotetext{
* Corresponding author. Fax: +886-2-29544672.

E-mail address: petecyy@ms19.hinet.net (Y.Y. Chiu).
}

the sheet, which has a thin layer of thermoplastic adhesive on the down-facing surface. After the new layer is bonded, a focused beam of $\mathrm{CO}_{2}$ laser incises the outline of the part. Laser power is adjusted to cut through only one layer of lamination. The unused material is left in place, however, diced with crosshatch into small pieces called "tiles" for easy removal. The iterative process of bonding and laser cutting is repeated until the construction of the final layer is completed. Once all layers have been laminated and cut, excess material is removed to expose the finished part.

Nevertheless, in the LOM process of paper lamination, there exist a lot of demerits and problems. For example, the LOM process adopts a "bond-then-cut" method where every layers of workpiece's region supported by waste material. By using such method, the waste material and workpiece would co-exist. Upon the completion of processing, labors are needed to de-cube the unnecessary waste material. This is also called de-cubing process. And in the process of de-cubing, not only would it waste the manpower and working hours, but also damage the workpiece itself. Besides, 
since the waste material surrounds with the workpiece, many geometrical parts (hollow part, vase-shaped part, thin wall part, etc.) cannot be fabricated by LOM process. As the waste material also has to be crosshatched during the laser cutting process and also consumes plenty of laser cutting energy.

In order to improve the problems of waste material removal process, Klosterman et al. [1] developed an adaptive crosshatches method. It uses the fine crosshatch laser cut to reduce the bonding strength at the contact area between the workpiece and waste material. However, as the fine crosshatch laser cut applied by this method is not the efficient method, it would induce an excessive laser processing path. Besides, the intensive laser cut will produce a very great amount of thermal effect, which would affect and restrict the bonding strength and precision of workpiece. Moreover, though the fine crosshatch method is applied, much time was still consumed on the waste material removal process. Apart from these, the laser cutting of waste material is not just an immense waste of energy, but also a considerable increase of time for laser cutting.

Thomas [2] proposes the method of "cut-then-bond" to solve the de-cubing problem encountered in LOM process. The principle of this method is to use laser to cut the $2 \mathrm{D}$ contour from slicing $3 \mathrm{D}$ CAD file on the working table. After that, by means of conveyance mechanism, the sheet-based material being cut is sent to another working table to carry out the pressing and bonding process. All the unnecessary waste material is removed during the process. Repeated the procedures 3D part is finished. Although the "cut-then-bond" method would not produce problem of waste material, the natural supporting feature of the waste material for LOM process is also lost. Moreover, the conveyance mechanism would not hold the sheet-based materials in a fixed position during the conveying process, which is also a serious problem.

As de-cubing process plays a very importance role in the LOM process, the adhesive invalidation approach is developed [3]. Making use of the control of laser processing path planning, it reduces the bonding strength between waste material and workpiece. After that, an adaptive crosshatch approach to improve working efficiency and to alleviate the effort involved in the de-cubing process is also developed [4]. In order to obtain a more extensive application of LOM in the aspect of complicated geometrical parts, as well as to achieve a higher efficiency of manufacturing and de-cubing process, the Bridge-LOM process and its associated building algorithm are proposed in this paper. It uses the bridge structure to connect all the 2D sections after slicing 3D CAD file. Based on the "cut-then-bond" method for lamination, the bridge structure not only provides a natural supporting feature, but also replaces the supporting function of waste material as comparing with the current LOM process. Besides, over $60-70 \%$ of waste materials would naturally be removed on-line. This proposed method intends to solve the problems of waste materials removing process in LOM process, also promotes the efficiency of processing as well, avoids the waste of unnecessary laser processing consumption, fabricates the complicated geometrical part that cannot be fabricated by the current LOM process, and expands the application area of LOM process. Furthermore, the metallic part and mold can be fabricated and their performances are investigated.

\section{Bridge-LOM process}

The proposed Bridge-LOM process adopts "feed-cut-press then adhesive spraying" method to laminate successive layers and a workpiece is finally produced. Fig. 1 shows the flow chart of Bridge-LOM process. The "feed" process adds new layer, "cut" the new layer by controlling laser path, "press" the layer with a pressing head, then the adhesive spraying process is done by nozzle. Repeat the procedures until the part is finished.

The frame structure of Bridge-LOM process approach is shown in Fig. 2. The layer material is a roll of sheet-based material. There are two rolls, a material supply roll which is used to feed the new layer, and a take-up roll which is used to collect the used layer, both are applied to control its forward movement and keep it at a certain tension. During the lamination process, the workpiece is placed on a table that lies beneath the layer material. The pressing head with vertical bonding movement is above the sheet of material, whereas a nozzle for spraying adhesive moves along $X-Y$ direction.

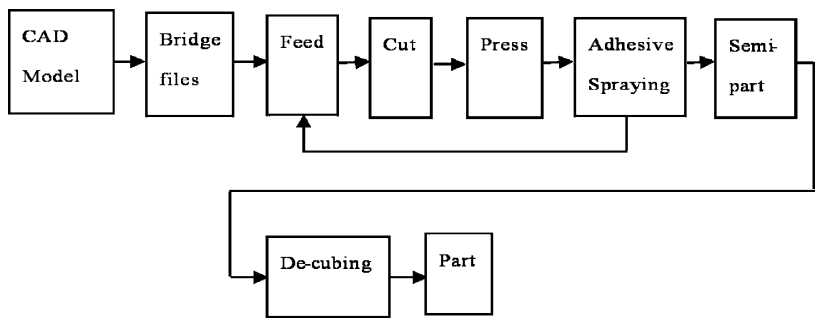

Fig. 1. Flow chart of Bridge-LOM process.

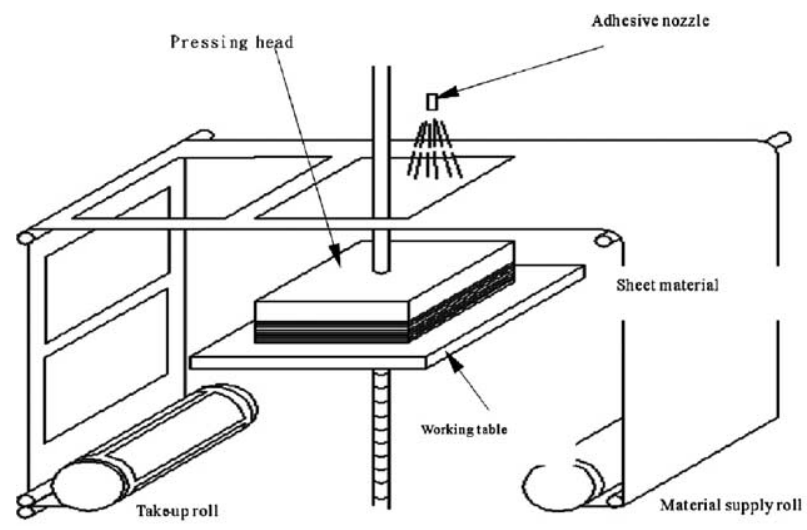

Fig. 2. Illustration of Bridge-LOM equipment. 


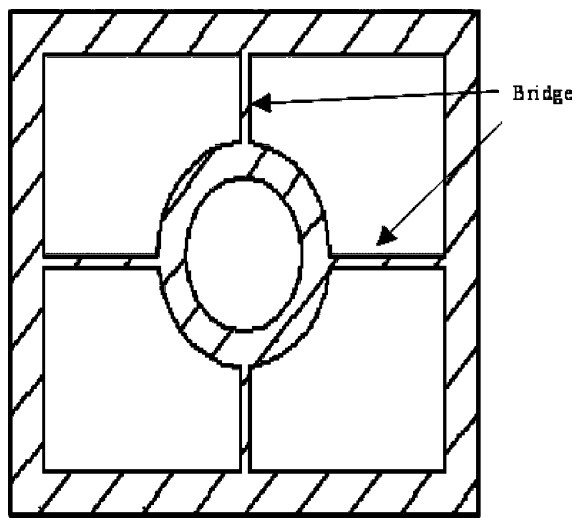

Fig. 3. Diagram of 2D contour after bridge, called bridge structure.

The detail description of the procedures of Bridge-LOM processing is stated as below:

Step 1. Make an extra outer frame to the 3D model established by CAD. A stack of 2D geometry contours is formed after slicing the 3D model. According to bridge building algorithm, the method will be specified in detail in the following section, convert it into a 2D geometry contours to the outer frame with bridge structure as shown in Fig. 3.

Step 2. Process and laminate the bridge-files accordingly. After the machine receives a bridge-file, make a complete laser cutting according to the contour of the file. One thing that is noteworthy: the most outer contour of each bridge-file has not been cut completely, in other words, the most outer contour still has a little bonding strength. After cutting the contour, the waste material inside the workpiece would drop completely, making an automatic de-cubing function. Finally, there are only three mutually connecting workpieces left each layer: outer frame, workpiece region and bridge. While the waste material is dropping, a simple sliding track mechanism used to receive the waste materials and then move them to the disposal bucket.

Step 3. After cutting the contour, the working table below moves upward and the pressing head above push down to bond with the previous layers together tightly that has just been cut.

Step 4. The pressing head and the working table move downward together, so that the cut layer and sheet-based material would separate completely. Also, this would ensure that this cut layer would bond with the previous layer tightly. In order to make the semi-cut contour separated from the outer contour of the cut layer, the edge of the upper pressing head can more or less be made into a knife shape. In doing so, when the pressing head moves downward, the knife shape at the outermost edge can create a cutting force to separate the contour smoothly.
Step 5. After the separation of the cut layer, the pressing head moves upward and retreats to the original position on top of the sheet-based material. The working table also retreats to the original position. Now the nozzle sprays adhesive as shown in Fig. 8.

Step 6. After the spraying of adhesive, the sheet-based material moves forward. The processing of one layer is then completed. Repeat this cycle to complete the whole processing.

Step 7. The workpiece will be exposed after the outer frame and bridge structure are removed.

\section{Bridge building algorithm approach}

The process developed under the principles of "cut-thenbond" has to face two important problems: support and position. Regarding the function of position, in order to ensure the proper bonding treatment, as well as holding a precise position between layers, the processes from cutting to bonding in the Bridge-LOM process should maintain the same $X-Y$ position. Therefore, worries of not having a fixed positioning would then be eliminated. Regarding the function of support, bridge has taken the place of the supporting function of waste material in the traditional LOM process. In other words, in the bridge building process, it moves upward from bottom up, naturally a supporting function towards the upper layer is reached. However, there is a phenomenal difference compared to Bridge-LOM and the traditional LOM process. The waste material support in the traditional LOM refers to the waste material existed in every part of the workpiece, exerting the support towards every part of the workpiece. As to the function of the bridge for Bridge-LOM process, apart from the bonding to the different contours of the same layer, it also builds bridge wherever support is required; so that the supporting function is formed to virtually anywhere the workpiece needs support. In this way, during the post-processing process of waste material removal, not only would the space occupied by the waste material of the bridge is less than that of the traditional LOM, but also the time spend on de-cubing is considerably reduced.

Bridge building algorithm approach is divided into three parts as follows.

\subsection{Workpiece's contour capture approach}

The purpose of workpiece's contour capture approach is to give every contour an attributive name, so as to follow up with the bridge building process. The idea of workpiece's contour capture approach is to "capture from the workpiece's outmost to the inner," with an order of A, B and C accordingly. For instance, the attributive name $\mathrm{B}_{m n}$, B refers to Bth layer, $m$ refers to the $m$ th group and $n$ refers to the $n$th contour of Bth layer. In the most outer frame, the contour of the inner layer is defined as "out-path". 
From workpiece's contour capture approach, it is known that the out-path area going inwards from $\mathrm{A}_{1}$ to $\mathrm{A}_{n}$ regions is waste material. The area from the region of $A_{1}$ to $A_{n}$ running inward to the region of $\mathrm{B}_{11}$ to $\mathrm{B}_{m n}$ is just the workpiece. Bearing such analogy in mind, the bridge can smoothly be established on the region of waste material to connect the different workpiece's region.

\subsection{Bridge building algorithm 1}

The purpose of the bridge building algorithm 1 is that while considering the $X-Y$ direction of the workpiece, allow mutual connection be possible between two contours of islands through the building of bridges, and in times of cutting, avoid the island from dropping together with the waste material. The categories of the bridge building algorithm 1 can be stated as below:

(1) Building bridge in the same sub-layer of the workpiece The contour attribute resulted from the workpiece's contour capture approach is used for connecting each of the island's contours of the same sub-layer together. The letter representing the contour attribute can judge whether it is of the same sub-layer. For example, $A_{1}$, $A_{2}$ and $A_{3}$ belong to the same sub-layer. The method of building bridge process stated as follow. First of all, find the centers of $A_{1}$ and $A_{2}$ islands, respectively. If draw a line come across these two centers, it will intersect with $A_{1}$ and $A_{2}$ contours, forming two intersecting points on different contour. On $A_{1}$ contour, take an intersecting point $b$ that is closer to $A_{2}$ center. On $A_{2}$ contour, take an intersecting point $c$ that is closer to $A_{1}$ center. Then the line $b c$ is obtained. Extend to both sides at half the width of the offset preset bridge. Immediately, the bridge connection is obtained as shown in Fig. 4. The purpose of this bridge building process in the same sub-layer of the workpiece is that prevent from the islands fall with the waste material due to their weights.

(2) Building bridge in the different sub-layer of workpiece's region

If workpiece's region still exists within a closed region that is called the different sub-layer here, it is necessary to combine two regions together to prevent

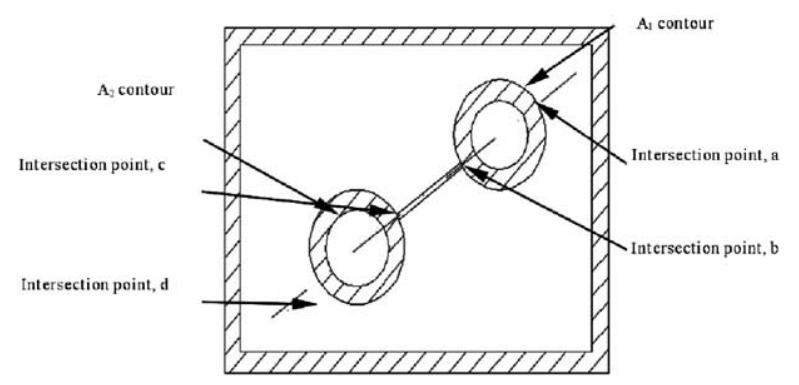

Fig. 4. Illustration of bridge building approach on the same sub-layer.

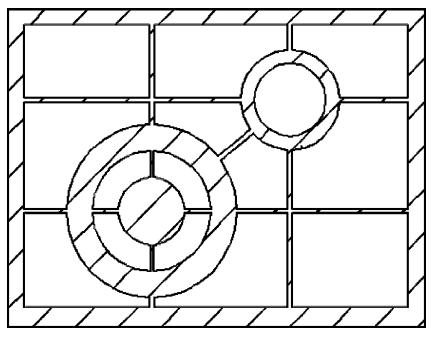

Fig. 5. Illustration of bridge building between sub-layer A contour and the outer frame.

dropping. The function of the bridge here is to connect the inner island with outer pocket within a closed region. Hence, the bonding strength of bridge between island and pocket will be formed.

(3) Make bridge between sub-layer A contours and the outer frame

Upon completion of bridge building and connection between two islands or pockets discussed earlier, the outermost layer of workpiece (i.e. sub-layer A contours) has to be connected with the outer frame. The method is to build a bridge along the $X$ - and $Y$-axis come across the centers of sub-layer A contours and the outer frame, as shown in Fig. 5.

\subsection{Bridge building algorithm 2}

The purpose of bridge building algorithm 2 is that consideration of the $Z$-axis of the workpiece, building bridges to prevent the creation of deflection caused by the weight of the workpiece's material:

\section{(1) 1st part of algorithm 2}

The difference of areas between the layers makes the following upper layer, after laminated, deflected at the protruding position because of insufficient support. Then incomplete bonding problems would appear in the lamination of the next layer in this case.

If the workpiece shown as in Fig. 6, there are small circular rods above and below, and an protruding shape in the middle. Upon completion of bridge building approach 1 , the bridges can only be built along $x$ - and $y$-axis. Therefore, the protrusion shape can be easily de-

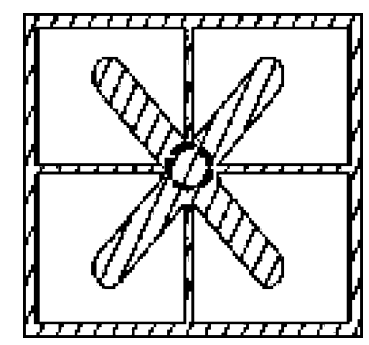

Fig. 6. Illustration of bridge building on the protruding rod according to bridge building algorithm 1 . 


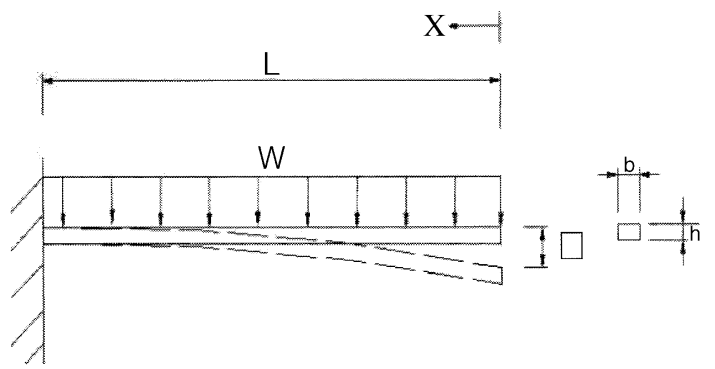

Fig. 7. Illustration of deflection viewed as suspended arm.

flected at $45^{\circ}$, and the next layer cannot be completely bonded during the lamination process.

Viewing these issues at the viewpoint of material kinematics as shown in Fig. 7, allow the edge of the small cylinder in the lower workpiece be the fulcrum of the suspended arm. Then the deflection $(\delta)$ of the free end is expressed as Eq. (1). If the acceptable $\delta_{\max }$ value is put in the equation, the acceptable $L_{\max }$ value can be obtained. Then take the more conservative $L_{\text {adaptive }}$ value as a basis for bridge building reference. Compare the actual $L$ value with the $L_{\text {adaptive }}$ value. When $L \geq$

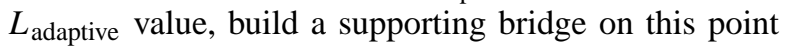
to prevent deflection.

$\delta=\frac{w L^{4}}{8 E I}$

This establishment is processed when there is a spatial difference between the upper and the lower layers. In order to make the established bridge to support the following laminated weight, a Boolean value should be established for reference. In this case, even though the Boolean value is true, build bridge on the several consecutive layers of the same point, so that the bridges there can be the support. By so doing, it would avoid deflection created from the following laminated weight.

(2) 2nd part of algorithm 2

Upon completion of cutting the sheet-based material, the contour difference of the same layer makes the more protruded contour bend seriously because of its own weight. Under this situation, wrinkles would appear during bonding process.

For example, the cross-section of the workpiece is entirely in the form of a protruding rod along $z$-axis. When the workpiece has gone through the process of laser cutting, the portion of protruding rod will bend downwards due to its own weight. When the pressing table undertakes bonding process downwards, wrinkles can be formed easily at the protrusion portion. Hence, a bridge still has to be built at the protruding rod for supporting. The bridge upon completion of building is also the same as 1st part of algorithm 2 .
Table 1

The comparisons of de-cubing time (min)

\begin{tabular}{lll}
\hline & $\begin{array}{l}\text { De-cubing time } \\
\text { of LOM }\end{array}$ & $\begin{array}{l}\text { De-cubing time } \\
\text { of Bridge-LOM }\end{array}$ \\
\hline Hollow bottle & 50 & 8 \\
Mobile phone & 55 & 22 \\
\hline
\end{tabular}

Table 2

The comparison of machining efficiency between Bridge-LOM and LOM process

\begin{tabular}{ll}
\hline & Bridge-LOM:LOM (time ratio) \\
\hline$T_{\text {prev }}$ & $\longrightarrow$ (slightly increase) \\
$t_{\text {bond }}$ & $\searrow$ (the same) \\
$L_{i} / v$ & $\searrow$ (greatly decrease) \\
$T_{\text {post }}$ & $\searrow$ (greatly decrease) \\
Total machining time & \\
\hline
\end{tabular}

\section{Conclusion}

The proposed Bridge-LOM process possesses the following advantages from the results of the experiment:

1. Bridge-LOM process and its associate bridge building algorithm approach can undertake on-line waste material removing of over $60-70 \%$ by its weight in the process. Afterwards, the time spent on de-cubing in the post-processing process can be greatly decreased by over $80 \%$ as shown in Table 1.

2. Through the bridge building approach, the process of crosshatching on the waste material as required in the original LOM process can be omitted. Not only can it save time on laser cutting, but also the consumption of laser power. It is, therefore, a great improvement to the processing efficiency, as shown in Table 2.

3. The on-line de-cubing feature of Bridge-LOM process can smoothly complete the fabrication of workpiece in geometrical patterns, such as hollow object, vase, etc. As for the fabrication of thin-wall workpiece, all one has to do is to remove the bridge during the post-processing process, so that the thin wall would not be damaged easily. Therefore, Bridge-LOM process can also fabricate the thin-wall part, the original LOM process still cannot build it.

4. Since Bridge-LOM process is extremely efficient in on-line de-cubing, it is suitable for the fabrication of

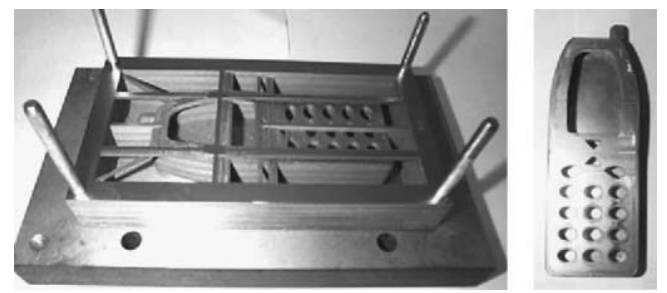

Fig. 8 . The metallic mobile phone prototype made by Bridge-LOM. 
hard material. The metal workpiece and mold are very good application for Bridge-LOM process, as shown in Fig. 8. There will be more researches in the filed. Investigate the heat-resistance situation, strength difference and number of injected parts after application of heat-resisting adhesive for bonding of metal molds.

\section{References}

[1] D. Klosterman, R. Chartoff, N. Osborne, G. Graves, Automated fabrication of monolithic and ceramic matrix composites via laminated object manufacturing (LOM), in: Proceedings of the Solid Freeform Fabrication Symposium for Electro-Machining, April 17-21, 1995, pp. 3-28.

[2] C.L. Thomas, Automating sheet-based fabrication: the ConveyedAdherent ${ }^{\mathrm{TM}}$ process, in: Proceedings of the Solid Freeform Fabrication Symposium, University of Texas at Austin, August 1996, pp. 281290.

[3] Y.Y. Chiu, Y.S. Liao, Laser path planning of burn-out zone for laminated object manufacturing (LOM) to improve the efficiency of waste removal process, in: Proceedings of the International Symposium for Electro-Machining (ISEM XIII), May 2001, pp. 813828.

[4] Y.S. Liao, Y.Y. Chiu, An adaptive crosshatch approach for laminated object manufacturing (LOM) process, Int. J. Prod. Res. 39 (15) (2001) 3479-3490. 